\title{
Differential effects of PD-L1 versus PD-1 blockade on myeloid inflammation in human cancer
}

Noffar Bar, ${ }^{1}$ Federica Costa, ${ }^{2,3}$ Rituparna Das, ${ }^{2}$ Alyssa Duffy, ${ }^{2}$ Mehmet Samur, ${ }^{4}$ Samuel McCachren, ${ }^{2}$ Scott N. Gettinger, ${ }^{1}$ Natalia Neparidze, ${ }^{1}$ Terri L. Parker, ${ }^{1}$ Jithendra Kini Bailur, ${ }^{2}$ Katherine Pendleton, ${ }^{2,5}$ Richa Bajpai, ${ }^{2}$ Lin Zhang, ${ }^{1}$ Mina L. Xu, ${ }^{6}$ Tara Anderson, ${ }^{1}$ Nicola Giuliani, ${ }^{3}$ Ajay Nooka, ${ }^{2}$ Hearn J. Cho, ${ }^{7}$ Aparna Raval, ${ }^{8}$ Mala Shanmugam, ${ }^{2,9}$ Kavita M. Dhodapkar, ${ }^{5,9}$ and Madhav V. Dhodapkar ${ }^{2,9}$

'Department of Medicine, Yale University School of Medicine, New Haven, Connecticut, USA. 'Department of Hematology/ Oncology, Emory University, Atlanta, Georgia, USA. ${ }^{3}$ Department of Medicine and Surgery, University of Parma, Parma, Italy. ${ }^{4}$ Department of Data Sciences, Dana-Farber Cancer Institute, Boston, Massachusetts, USA. ${ }^{5}$ Department of Pediatrics, Children's Healthcare of Atlanta, Emory University, Atlanta, Georgia, USA. 'Department of Pathology, Yale University School of Medicine, New Haven, Connecticut, USA. ${ }^{7}$ Mount Sinai Medical Center, New York, New York, USA ${ }^{8}$ Oncology Biomarker Development, Genentech, South San Francisco, California, USA. ${ }^{9}$ Winship Cancer Institute, Emory University, Atlanta, Georgia, USA.

BACKGROUND. PD-1 and PD-L1 have been studied interchangeably in the clinic as checkpoints to reinvigorate T cells in diverse tumor types. Data for biologic effects of checkpoint blockade in human premalignancy are limited.

METHODS. We analyzed the immunologic effects of PD-L1 blockade in a clinical trial of atezolizumab in patients with asymptomatic multiple myeloma (AMM), a precursor to clinical malignancy. Genomic signatures of PD-L1 blockade in purified monocytes and T cells in vivo were also compared with those following PD-1 blockade in lung cancer patients. Effects of PD-L1 blockade on monocyte-derived DCs were analyzed to better understand its effects on myeloid antigenpresenting cells.

RESULTS. In contrast to anti-PD-1 therapy, anti-PD-L1 therapy led to a distinct inflammatory signature in CD14+ monocytes and increase in myeloid-derived cytokines (e.g., IL-18) in vivo. Treatment of AMM patients with atezolizumab led to rapid activation and expansion of circulating myeloid cells, which persisted in the BM. Blockade of PD-L1 on purified monocyte-derived DCs led to rapid inflammasome activation and synergized with CD40L-driven DC maturation, leading to greater antigen-specific $\mathrm{T}$ cell expansion.

CONCLUSION. These data show that PD-L1 blockade leads to distinct systemic immunologic effects compared with PD-1 blockade in vivo in humans, particularly manifest as rapid myeloid activation. These findings also suggest an additional role for PD-L1 as a checkpoint for regulating inflammatory phenotype of myeloid cells and antigen presentation in DCs, which may be harnessed to improve PD-L1-based combination therapies.

TRIAL REGISTRATION. NCT02784483.

FUNDING. This work is supported, in part, by funds from NIH/NCI (NCI CA197603, CA238471, and CA208328).

Copyright: () 2020, American Society for Clinical Investigation.

Submitted: April 9, 2019

Accepted: May 13, 2020

Published: June 18, 2020

Reference information: /CI Insight. 2020;5(12):e129353.

https://doi.org/10.1172/jci.

insight.129353.

\section{Introduction}

Antibody-mediated blockade of PD-1 or PD-L1 has led to tumor regression and improved survival in a subset of patients with diverse tumor types (1). PD-L1-expressing tumor cells and antigen-presenting cells (APCs) engage PD-1+ $\mathrm{T}$ cells, leading to T cell dysfunction. In view of the dominant role of T cells in tumor immunity, blockade of PD-1 or PD-L1 has been studied interchangeably in clinical cancer immunotherapy 
as a strategy to activate T cells. However, both molecules have alternate ligands/receptors; it has also been suggested that PD-L1 can act as a receptor to back-transmit signals into T cells (2) and tumor cells (3). PD-L1 is constitutively expressed on a subset of myeloid APCs, including DCs, and prior studies in murine models have suggested a functional role for PD-L1 in myeloid cells or DCs (4-6). Direct comparison of signaling pathways altered in vivo following PD-1 or PD-L1 blockade in T cells and APCs in humans are limited and may help optimal design of combination therapies with these antibodies (Figure 1A). While the PD-L1 axis has been extensively studied in the context of immunotherapy of established cancer, data about the effects of PD-L1 blockade on premalignant states are limited.

Multiple myeloma (MM) is a common hematologic malignancy, which is preceded in all cases by well-defined precursor states, monoclonal gammopathy of undetermined significance (MGUS), and asymptomatic MM (AMM) (7). In spite of major therapeutic advances, there is an unmet need to achieve durable unmaintained responses in this malignancy, prompting the need to pursue strategies to engage long-term immunologic memory against tumor cells. Antibody-mediated blockade of PD-1 as a single agent did not lead to tumor regression in relapsed MM (8). Prior studies have demonstrated immune recognition of preneoplastic MGUS cells by T cells $(9,10)$. In a prospective trial, the presence of preexisting $\mathrm{T}$ cell immunity to an embryonal stem cell antigen SOX2 was associated with reduced risk of progression to clinical MM (11). MM tumor cells commonly express PD-L1, and the expression of PD-L1 on MGUS/ AMM cells correlated with an increased risk of transformation to clinical malignancy $(11,12)$. These considerations prompted us to initiate a clinical trial of single-agent anti-PD-L1 antibody (atezolizumab) in patients with AMM (Figure 1B).

\section{Results}

Prior studies have shown that therapy with anti-CTLA-4, anti-PD-1, or combination leads to distinct genomic signatures in purified human T cells and monocytes in vivo (13). In order to compare the genomic and proteomic profiles of anti-PD-1 and anti-PD-L1 therapies, we isolated T cells and CD14 ${ }^{+}$monocytes from peripheral blood before and after anti-PD-L1 therapy in patients with advanced non-small cell lung cancer and analyzed changes in gene expression using Affymetrix HTA v2.0 array (Figure 1A). In direct contrast to prior studies with anti-PD-1 therapy, which predominantly leads to gene expression changes in T cells (13), anti-PD-L1 therapy led to dominant gene expression changes in CD14 ${ }^{+}$ monocytes (Figure 2A). Importantly, changes in gene expression following anti-PD-L1 therapy in both $\mathrm{T}$ cells and monocytes were nonoverlapping with those observed following anti-PD-1 therapy (Figure 2A). Top differentially expressed genes (DEG) in myeloid cells following PD-L1 blockade included inflammation-associated genes such as heparin-binding EGF-like growth factor (HBEGF), thrombospondin-1 (THBS), IL-1 $\beta$, CXCL1/GRO $\alpha$, CXCL2, and NLRP3 (Figure 2B). Pathway analysis of DEGs $(q<0.01)$ in monocytes revealed pathways related to inflammation and inflammasome-associated cytokines (IL1 and IL-18) (Supplemental Table 1; supplemental material available online with this article; https:// doi.org/10.1172/jci.insight.129353DS1). In order to further validate these data in the context of samples analyzed together and determine if these signals were derived from only a subset of monocytes, we analyzed purified monocytes from patients before and after anti-PD-1/PD-L1 therapy using single cell RNA sequencing (RNA-seq). These data demonstrate that early changes in myeloid cells were again more prominent following PD-L1 blockade (Figure 2C) and involved nearly all classical monocytes (Figure 2D). Changes in gene expression in these monocytes were similar to those in earlier studies (Supplemental Figure 1A) and also revealed pathways consistent with myeloid activation (Supplemental Figure 1B). Analysis of sera before and after therapy demonstrated that, while both therapies led to an increase in IP-10 as a marker of immune activation, increases in serum IL-18, GRO $\alpha$, IFN- $\alpha 2$ typically derived from myeloid cells and sCD40L, are only observed following anti-PD-L1 therapy (Figure 3, A-E). Taken together, these data demonstrate that systemic immunologic changes following anti-PD-L1 therapy are surprisingly distinct from that following anti-PD-1 therapy, both at genomic and proteomic levels - in particular, with rapid activation of inflammation-associated genes in monocytes.

Expression of PD-L1 was previously correlated with the risk of progression to MM (11). In order to evaluate the potential of targeting the PD-L1 axis to prevent MM, we enrolled AMM patients in a pilot trial of single-agent atezolizumab. The trial was closed prematurely based on FDA guidance after enrollment of only 2 patients due to safety concerns emerging in 2 clinical trials of pembrolizumab and lenalidomide in MM. Clinical data from these patients are summarized in Supplemental Table 2. Both patients had stable 
A

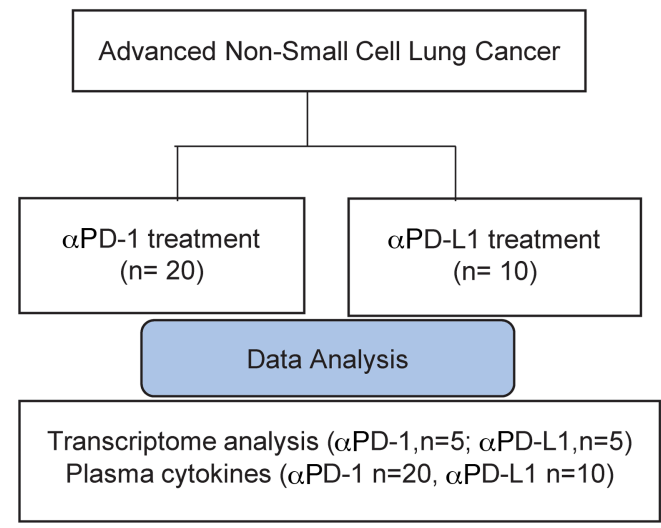

B

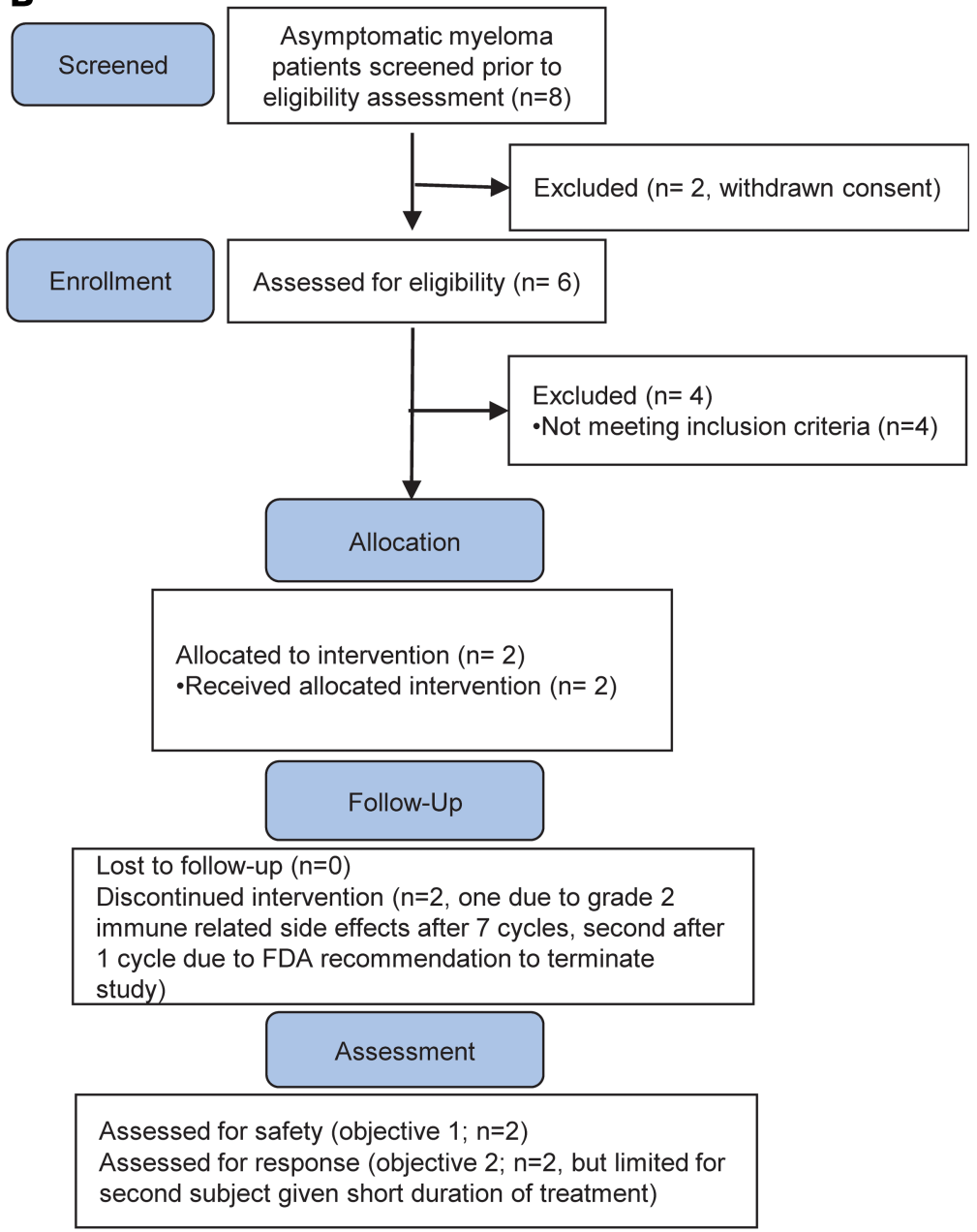

Figure 1. CONSORT flow diagram for non-small cell lung cancer and asymptomatic myeloma clinical trials. (A) Diagram reporting the distribution of $\alpha \mathrm{PD}-\mathrm{L} 1$ and $\alpha$ PD-1 treatment in patients with non-small cell lung cancer and subsequent data analysis. (B) Flow diagram reporting the process of screening, enrollment, allocation, follow-up, and assessment through the phases of the clinical trial of single-agent atezolizumab in patients with asymptomatic myeloma.

disease at the time of study closure, after receiving 7 and 1 cycles, respectively, and remain progression-free off therapy with 23-and 18-month follow-up. Patient 1 developed grade 2 endocrinopathy with hypothyroidism and adrenal insufficiency after 7 cycles. Incidentally, this patient also experienced remission of prior gluten intolerance after enrolling in the study. Serial analysis of peripheral blood samples by mass cytometry revealed an early increase in blood monocytes and a decline in B cells in both patients, detected at 15 days after initiation of therapy (cycle 1 day 15; C1D15) (Figure 4A and Supplemental Figure 2A). Phenotypic analysis revealed an increase in $C D 16^{+} C D 40^{+} \mathrm{HLADR}^{\text {hi }}$ monocytes (Figure $4 \mathrm{~B}$ ). In order to validate these findings in an independent data set, we analyzed early changes in blood monocytes from another clinical trial in MM (NCT02431208), wherein a cohort received single-agent atezolizumab. These data also corroborate our prior studies and demonstrate a similar pattern of rapid increase in circulating activated monocytes in vivo (Figure 4C). Taken together, data from both lung cancer and MM patients show that PD-L1 blockade leads to early activation of myeloid cells with a transient increase in activated circulating monocytes in vivo.

Evaluation of $\mathrm{T}$ cells in AMM patients treated with atezolizumab revealed an early increase in circulating $\mathrm{CD}^{+}$and $\mathrm{CD} 4^{+}$memory $\mathrm{T}$ cells detectable by C1D15 (Figure 5A and Supplemental Figure 2B). Single cell mass cytometry revealed proliferation of $\mathrm{CD} 8^{+}$and $\mathrm{CD} 4^{+}$effector memory (Tem) compartment, as well $\mathrm{CD} 8^{+}$central memory (Tcm) compartment, manifest as upregulation of Ki-67 (Figure $5 \mathrm{~B})$. In prior studies, we have shown that SOX2 is a common antigenic target of $\mathrm{T}$ cells in MGUS (10). Evaluation of antigen-specific T cells at C1D15 also revealed an increase in antigen-reactive IP10 
A

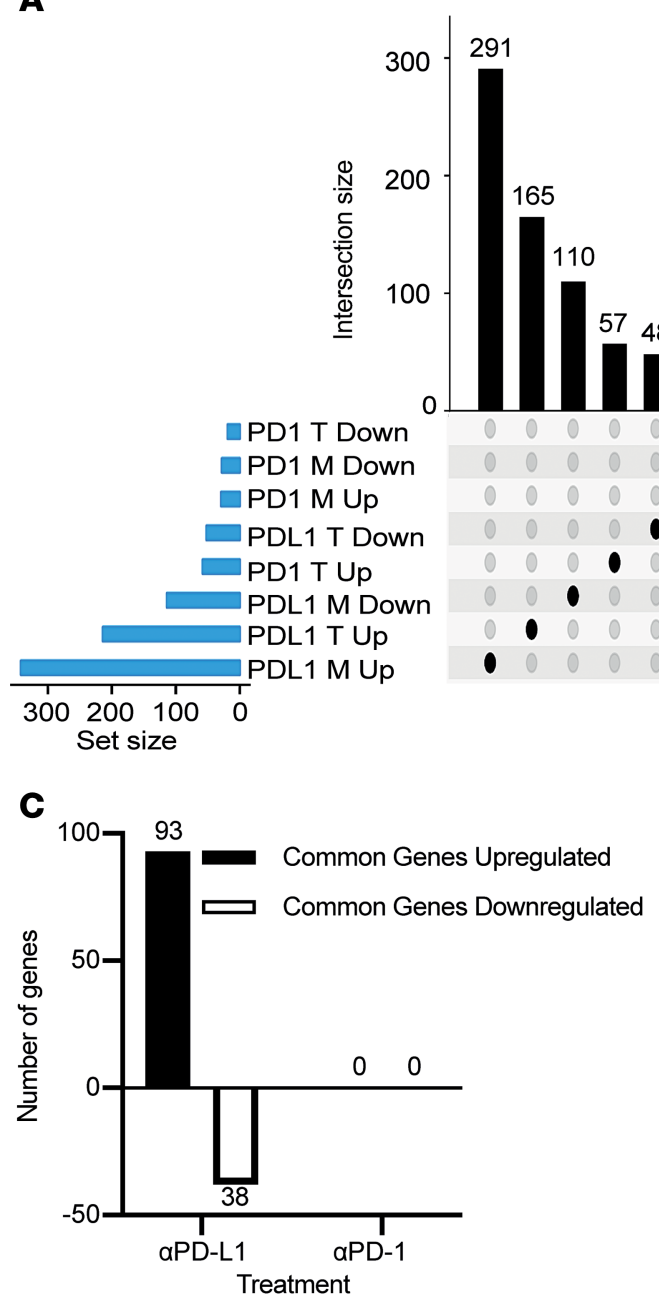

B

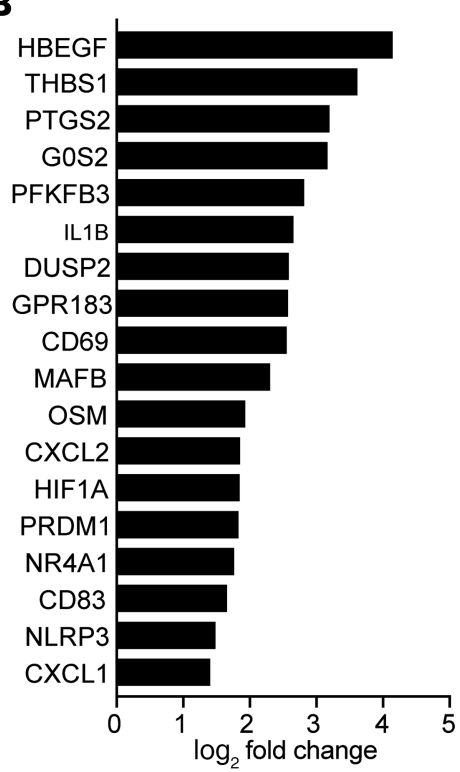

D

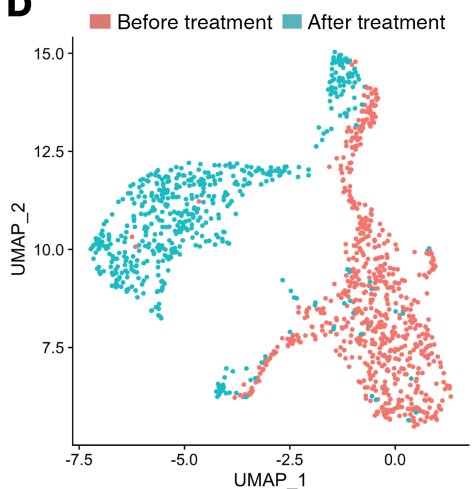

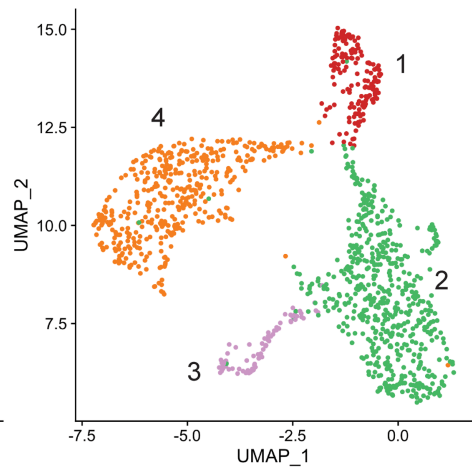

Figure 2. PD-L1 blockade leads to distinct transcriptomic changes in circulating monocytes and T cells. RNA was extracted from magnetic bead isolated $\mathrm{CD}_{14}{ }^{+}$monocytes and $\mathrm{CD}^{+} \mathrm{T}$ cells from patients with lung cancer before and after therapy with either anti-PD-L1 (atezolizumab; $n=5$ ) or anti-PD-1 (nivolumab; $n=6$ previously published; ref. 10) and analyzed using affymetrix human transcriptome array 2.0. (A) Distribution of differentially regulated genes upregulated and downregulated in monocytes and T cells following therapy with anti-PD-L1 or anti-PD-1. (B) Differentially regulated genes in monocytes following therapy with anti-PD-L1 (selected from top 50 differentially regulated genes). (C) Single cell RNA sequencing was performed before and after therapy with either anti-PD-L1 $(n=3)$ or anti-PD-1 $(n=4)$. Figure shows the number of shared differentially expressed (Wilcoxon rank-sum with Bonferroni's correction, $P<0.05)$ genes after versus before treatment between all anti-PD-L1 treated monocytes and all anti-PD-1-treated monocytes. (D) Uniform manifold approximation and projection (UMAP) plots of monocytes from single cell RNA sequencing of anti-PD-L1 monocytes before and after treatment (left panel: blue, after treatment; red, before treatment) and monocyte groups identified by unsupervised clustering (right panel). Cluster 1 represents CD16+ monocytes; clusters 2, 3, and 4 represent CD16- monocytes.

production following stimulation with SOX2-peptide library (Figure 5C). However, therapy-induced changes in circulating $\mathrm{T}$ cells were transient and returned to baseline by cycle 2 . Although the number of total B cells declined, therapy was also associated with an increase in the CD21 $1^{\text {lo }} \mathrm{B}$ cell subset implicated in autoimmunity (ref. 14 and data not shown). Analysis of serum cytokines also revealed early but transient changes in inflammatory cytokines (IL-18, IP-10, GRO $\alpha$, and TNF- $\alpha$ ), which returned closer to baseline by cycle 2 (Supplemental Figure 3). Together, these data show that atezolizumab leads to rapid but only transient systemic immune activation in vivo in AMM patients.

Evaluation of posttreatment BM specimens was planned after the completion of 2 cycles and, therefore, was obtained in only 1 patient. Posttreatment BM revealed a decline in T and B cells but clear increase in the proportion of $\mathrm{CD} 14^{+}$myeloid cells (Figure 6, A and B), which also exhibited some evidence of activation manifest with upregulation of HLA-DR (Figure 6C). Although reduced in number, BM memory $\mathrm{T}$ cells from posttreatment biopsies did demonstrate an increase in granzyme and T-bet relative to baseline samples, particularly within the Tem subset (Figure 6D). 

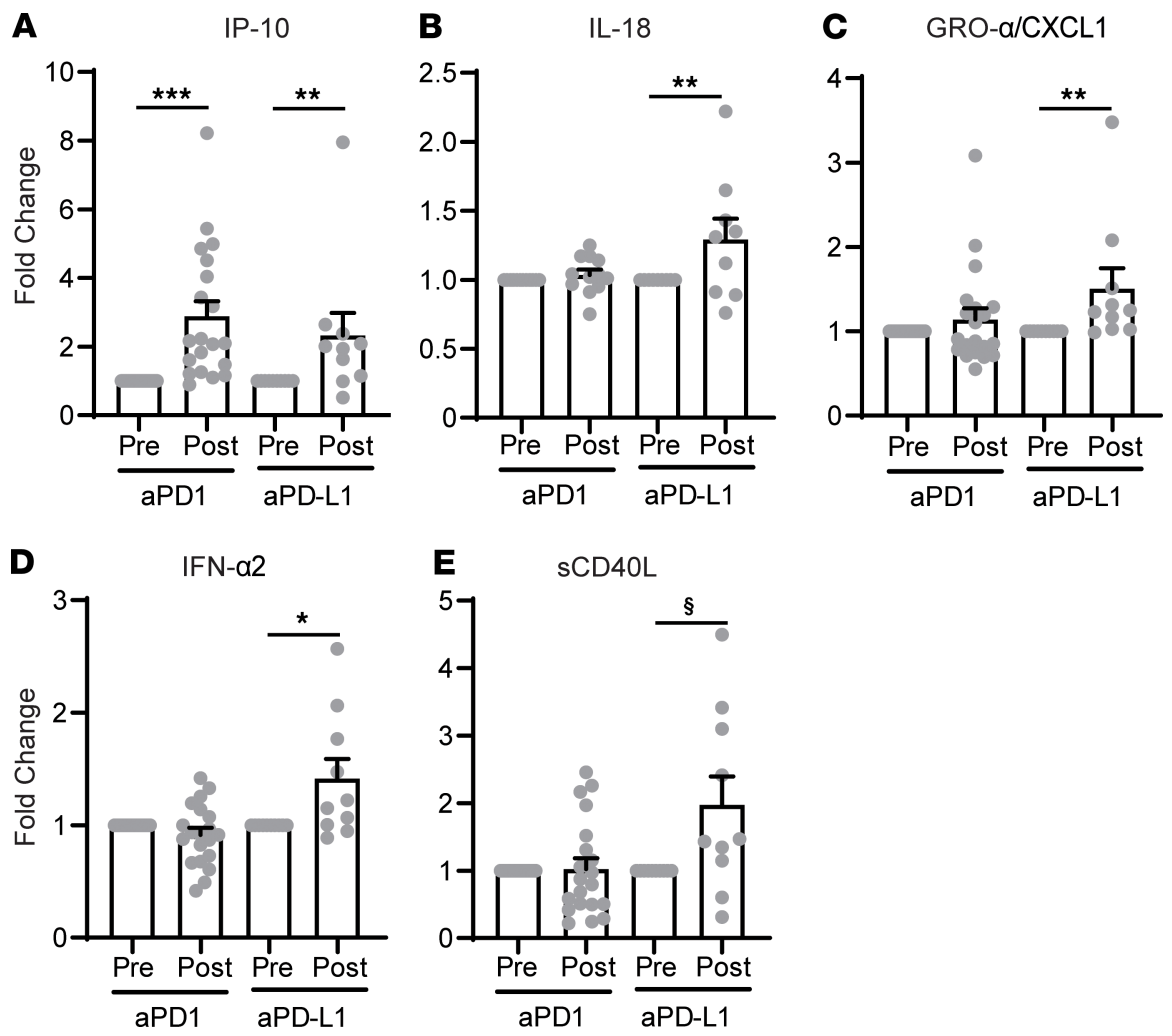

Figure 3. PD-L1 blockade leads to distinct plasma cytokine profiles. Plasma collected before and after therapy with anti-PD-L1 $(n=10)$ or anti-PD-1 ( $n=20$, as previously published; ref. 10) was analyzed using Luminex multiplex/ELISA. Figure shows changes in plasma IP-10 (A), IL-18 (B), CRO- $\alpha$ /CXCL1 (C), IFN- $\alpha 2$ (D), and sCD40L (E) following therapy with anti-PD-L1 or anti-PD-1. $\left({ }^{*} P<0.05,{ }^{* *} P<0.01,{ }^{* *} P<0.001,{ }^{s} P=0.06\right.$ by Mann-Whitney $U$ test).

In order to understand the observed changes in immune cells in further detail, we analyzed single cell transcriptomes of circulating as well as BM mononuclear cells. In the BM, single cell RNA-seq (sc-RNA-seq) identified 6 major T/NK cell clusters (Figure 7, A and B). Of these, the proportion of T cell clusters 0 and 3 declined in the posttreatment biopsy, consistent with reduction in $\mathrm{T}$ cells detected by mass cytometry. In contrast, there was an increase in the proportion of cells in several myeloid clusters, including classical CD $14^{+}$myeloid cells (clusters 5), CD16 ${ }^{+}$myeloid cells (cluster 7), and DCs (cluster 8). Pathway analysis of DEGs in these clusters demonstrated an increase in TNF- $\alpha$ signaling and IFN- $\alpha$ response in myeloid cells, as well as other cell types, consistent with evidence of inflammatory signaling in posttreatment BM (Supplemental Table 3). sc-RNA-seq analysis of paired blood samples from baseline and C1D15 from both patients also demonstrated systemic changes in gene expression, particularly in cluster 2 (myeloid cells) and cluster 5 (B cells), consistent with prior results using mass cytometry (Supplemental Figure 4 and Supplemental Table 4). Pathway analysis revealed an enrichment of IFN response and inflammation-associated pathways after therapy in several major circulating cell types ( $\mathrm{T}$ cells, B cells, monocytes, and NK cells), consistent with systemic immune activation and changes in serum cytokines at this time point (Supplemental Table 4).

Among myeloid cells, DCs constitutively express high levels of PD-L1. Prior studies have mostly focused on effects of PD-L1 blockade in the context of DC-T cell interactions (1). In order to test if PD-L1 may directly impact the biology of human monocyte-derived DCs (Mo-DCs) independent of DC-T cell interactions, we cultured purified Mo-DCs with anti-PD-L1 antibodies. Culture of Mo-DCs with anti-PD-L1, but not anti-PD-1, led to modest increases in CD80 and CD83 as markers of DC maturation (Figure $8 \mathrm{~A}$ ). This was associated with an increase in the secretion of several inflammatory cytokines - notably, IL-6, IL-8, TNF- $\alpha$, and IL-1 $\beta$ - in the culture supernatants (Figure 8B), as well as rapid (within 4 hours) activation of caspase-1 (Figure $8 \mathrm{C}$ ) and changes in cellular energetics associated with DC maturation, manifest as an increase in spare respiratory capacity (Figure 8D). In the setting of DC-T cell interaction, CD40L-mediated licensing of DCs is a critical regulator of antigen presentation (15). 
A

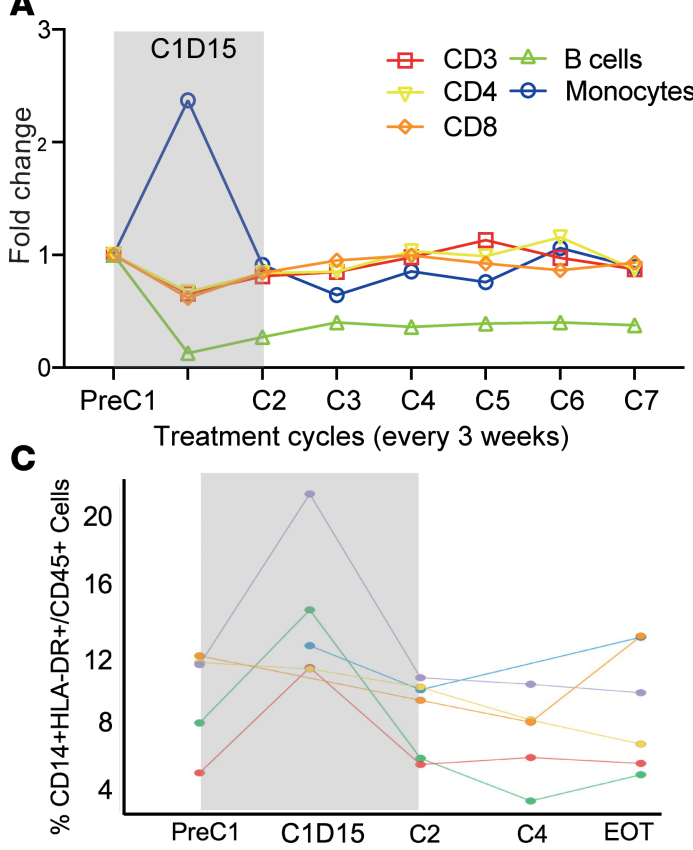

B
PT1

PT2

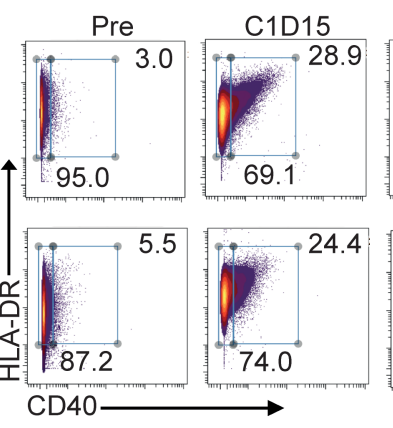

C1D21

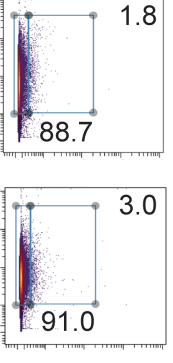

PT1
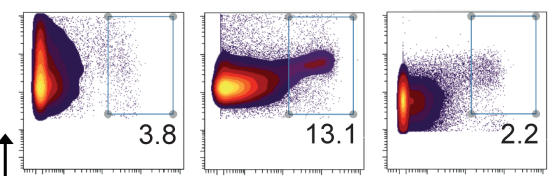

PT2
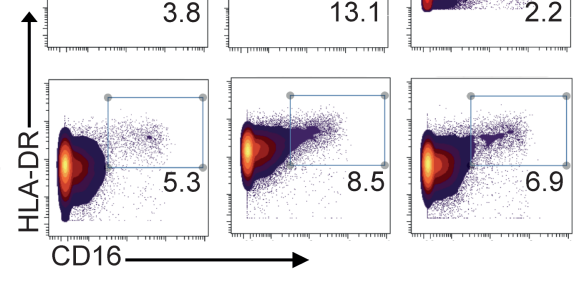

Figure 4. Changes in circulating immune cells following therapy with anti-PD-L1 in asymptomatic myeloma (AMM). PBMCs isolated from blood of AMM patients before therapy (PreC1) and following therapy with atezolizumab on day 15 (C1D15), as well as before cycles 2-7 (C2-C7) were analyzed using single cell mass cytometry or CyTOF. (A) Changes in circulating $\mathrm{CD}^{+}, \mathrm{CD}^{+}, \mathrm{CD}^{+} \mathrm{T}$ cells, monocytes, and $\mathrm{B}$ cells. Data are shown as fold change compared with pretherapy (PreC1) levels. (B) Expression of CD40, HLA-DR, and CD16 on circulating monocytes before therapy (Pre), on day 15 following first dose (C1D15), and before second dose of atezolizumab (C1D21) in 2 different patients (PT1 and PT2). (C) Changes in circulating monocytes in MM patients receiving atezolizumab in another clinical trial (NCTO2431208). Each line represents an individual patient. EOT, end of therapy.

Therefore, we examined the impact of PD-L1 blockade on DC maturation following suboptimal concentration of CD40L. PD-L1 blockade led to an increase in CD40L-driven DC maturation, as detected by the expression of CD80 and CD83 (Figure 9, A and B), but also greater expansion of influenza-matrix peptide-specific (Flu-MP-specific) T cells by Flu-MP-loaded DCs (Figure 9C). Expression of PD-L1 in human Mo-DCs can vary in a donor-dependent fashion. Expression of PD-L1 on DCs correlated with the observed synergy for DC maturation with CD40L and atezolizumab (Supplemental Figure 5A). In order to further evaluate the effects of PD-L1 blockade on naturally occurring BM myeloid cells, we cultured these cells with atezolizumab. Consistent with our in vivo data, atezolizumab also led to an increase in $\mathrm{CD}_{16}{ }^{+} \mathrm{HLADR}{ }^{+} \mathrm{CD} 14^{+} \mathrm{BM}$ myeloid cells in culture (Supplemental Figure 5B).

\section{Discussion}

Together, these data demonstrate that PD-L1 blockade leads to a distinct genomic signature characterized by early activation and expansion of myeloid compartment in vivo. Therefore, while both PD-1 and PD-L1 blockade share well-studied effects in terms of reinvigoration of T cells, PD-L1 blockade also unleashes an underappreciated myeloid inflammatory checkpoint in vivo in humans. These findings are also consistent with recent data on PD-L1-mediated regulation of macrophage activation and proliferation in PD-L1deficient mice (16).

Understanding differences between PD-1 and PD-L1 blockade will be essential for optimal design of rational combination therapies with these approaches and may differ for each of these targets. Differential effects of PD-1 versus PD-L1 blockade on myeloid cells in vivo may also help explain why PD-L1 expression on myeloid cells better predicts responsiveness to PD-L1 than PD-1 blockade in the clinic (17). PD-L1 blockade of human DCs led to rapid activation of caspase-1/inflammasome, with upregulation of NLRP3 and inflammasome-dependent cytokines such as IL-18. Inflammasome activation plays a complex and context-dependent protumor/antitumor role in tumor immunity (18). Activation of NLRP3 inflammasome in DCs was shown to be critical for induction of adaptive immunity to dying tumor cells following 
A

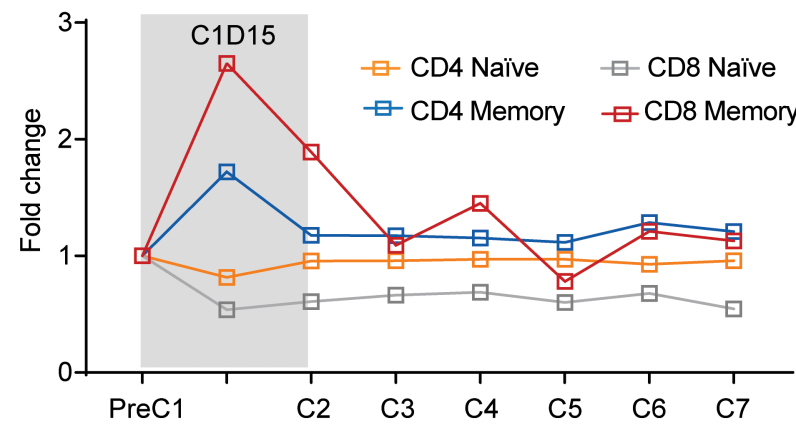

B
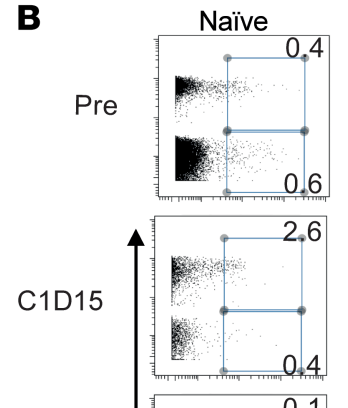

C1D21

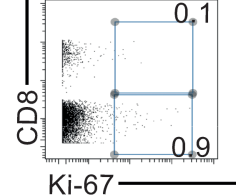
TCM

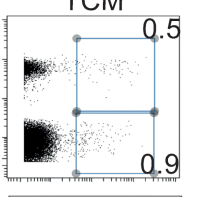
TEM
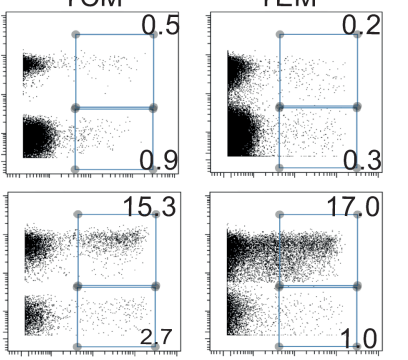

C

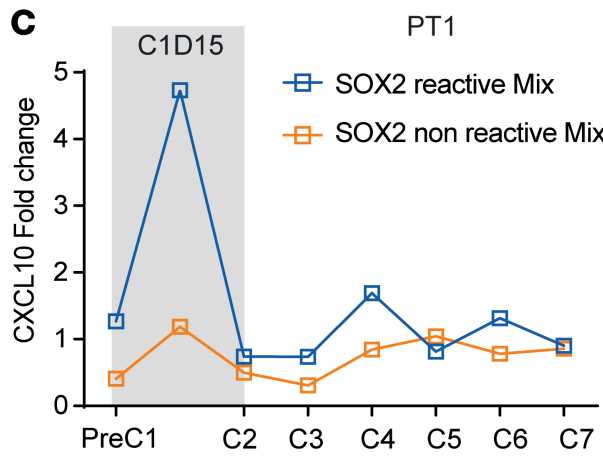

PT2

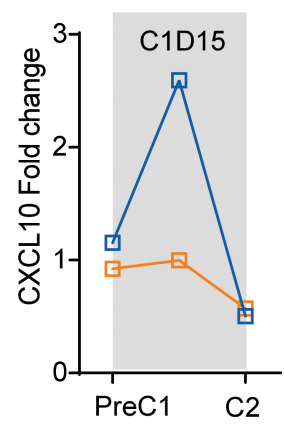

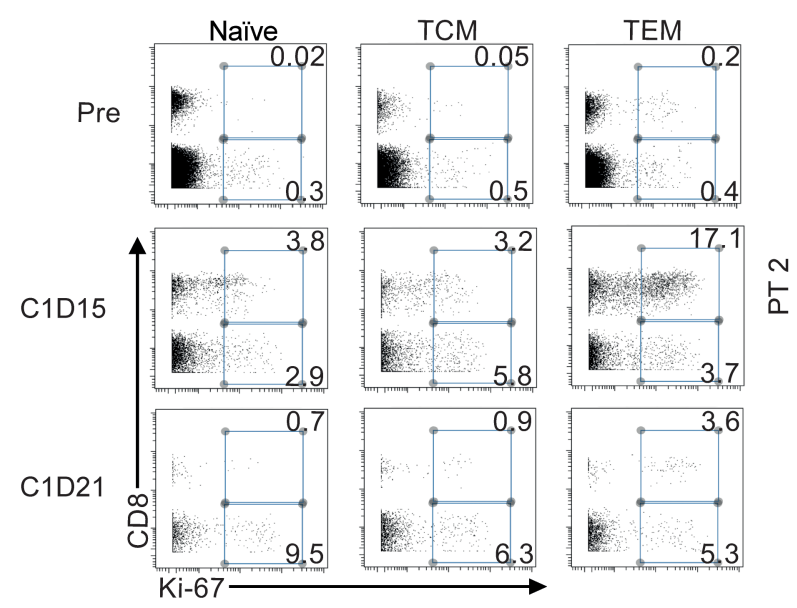

Figure 5. Changes in circulating T cells following therapy with anti-PD-L1 in asymptomatic myeloma (AMM). PBMCs isolated from blood of AMM patients before therapy (PreC1) and following therapy with atezolizumab on day 15 (C1D15), as well as before cycles 2-7 (C2-C7), were analyzed using single cell mass cytometry or CyTOF. (A) Changes in CD4 and CD8 naive and memory T cells during therapy with atezolizumab. Data are shown as fold change compared with levels before starting therapy (PreC1). (B) Ki-67+ proliferating naive (CCR7+RO-), central memory (Tcm; CCR7+RO+), and effector memory (Tem; CCR7-RO+) T cells before (Pre), 15 days following start of therapy (C1D15), and before cycle 2 (C1D21) of therapy with atezolizumab. Figure shows data from 2 separate patients. (C) PBMCs obtained pre therapy (PreC1), 15 days after starting therapy (C1D15), or before cycles 2-7 (C2-C7) were evaluated for the presence of SOX2-specific T cell reactivity using overlapping peptides encompassing the entire SOX2 antigen as previously described (10). Figure shows SOX2 T cell reactivity in the 2 patients. Data reported as fold change compared with before therapy (PreC1) for SOX2 reactive submix versus nonreactive mix as control.

chemotherapy (19). Therefore, PD-L1 may play an important role in the afferent arm of tumor-immunity cycle in regulating antigen presentation. The finding that PD-L1 blockade may enhance CD40L/T cell-mediated DC maturation may provide the rationale for combinations of PD-L1 blockade with agents targeting agonistic $\mathrm{CD} 40$ signaling.

Effect of PD-L1 blockade on myeloid cells in vivo could, however, also have potential undesired effects. Enrichment and activation of myeloid cells following PD-L1 blockade may lead to T cell exclusion and resistance to PD-L1 blockade in myeloid-rich tumors (20). Effects on myeloid cells could also have contributed to the lack of persistent $\mathrm{T}$ cell activation following atezolizumab that we observed in AMM patients, and this suggests that combinations with therapies that inhibit enrichment of myeloid compartment may be explored to improve PD-L1 blockade. Recent studies have also suggested the potential for myeloid cells to mediate hyperprogression in some tumors (21); prior studies have, indeed, shown the capacity of myeloid cells to promote MM growth $(22,23)$.

While the small number of patients treated due to regulatory issues limits interpretation, the correlative immunologic data in this earlier stage do demonstrate the feasibility to achieve immune activation in the tumor bed. The bar for acceptable complications is lower in this setting than in clinical myeloma. Therefore, careful selection of patients more likely to respond to immune therapies would be important for future investigations in immune-prevention based on checkpoint blockade. In this regard, recent studies show that loss of stem-like and marrow-resident $\mathrm{T}$ cells is an early feature of MM, which may restrict the efficacy of checkpoint blockade in this setting (24). It is, however, notable that the finding of increase in inflammasome-dependent cytokines such as IL-18 has been prominently demonstrated in large cohorts of patients treated with anti-PD-L1 but not anti-PD-1 antibodies, which is consistent with our data (17). 
A

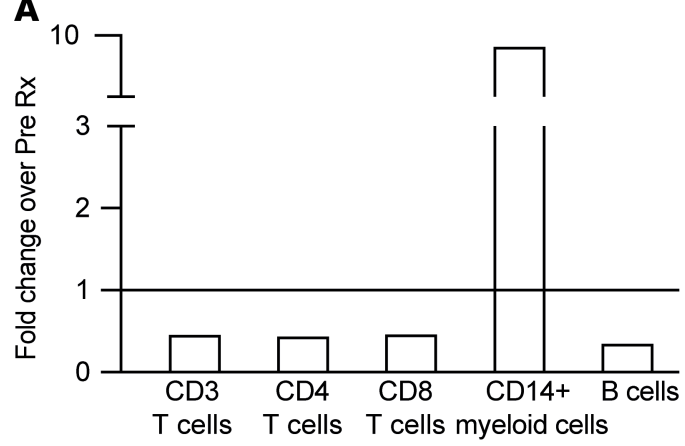

B

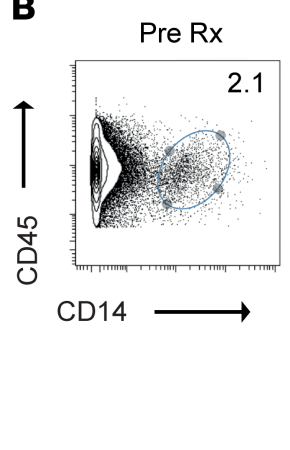

C

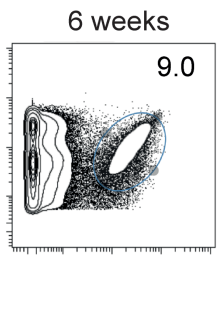

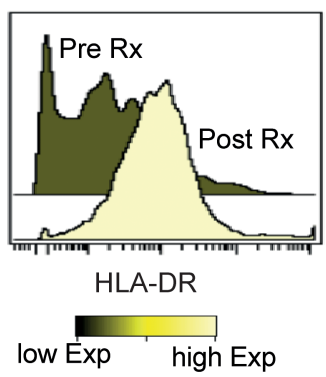

D
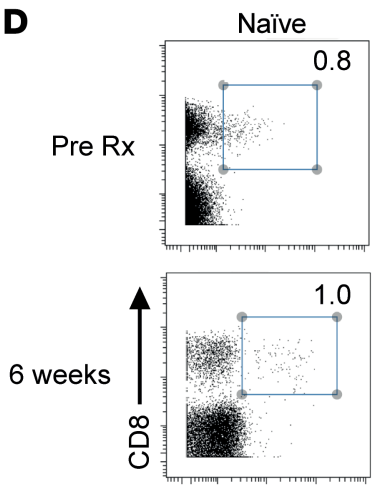

Granzyme
TCM
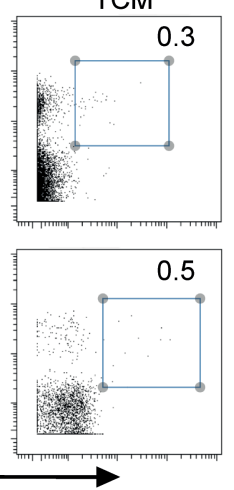

TEM
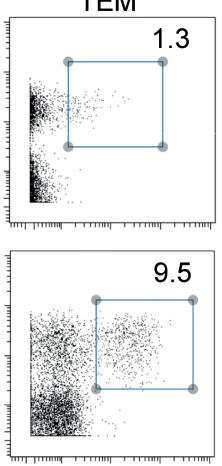
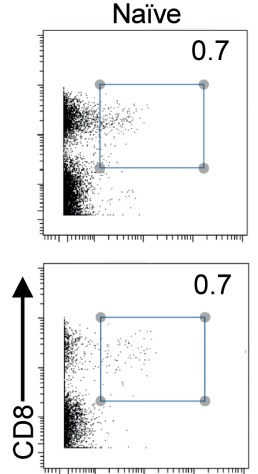

T-bet
TCM
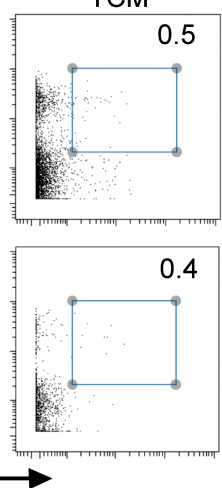

TEM
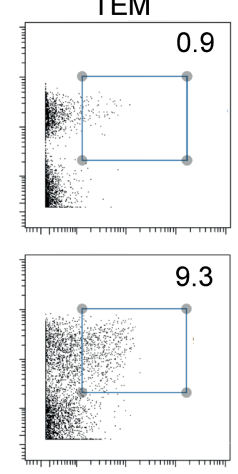

Figure 6. Changes in monocytes and effector T cells in BM following therapy with anti-PD-L1 in AMM by mass cytometry. BM was collected from AMM patient before and after 2 cycles ( 6 weeks) of therapy with atezolizumab. Mononuclear cells were isolated and analyzed using single cell mass cytometry, as well as single cell RNA sequencing. (A) Bar graph shows changes in CD3, CD4, CD8 T cells, CD14+ myeloid cells and B cells at 6 weeks following therapy with atezolizumab. (B) Changes in CD14+ myeloid cells. (C) Histogram showing changes in HLA-DR expression in CD14 ${ }^{+}$myeloid cells following therapy with atezolizumab. (D) Proportions of granzyme and T-bet positive naive, Tcm, and Tem cells in the marrow before start of therapy, as well as 6 weeks following therapy with atezolizumab.

An important limitation of these data is the small number of patients studied, due to early closure of the trial linked to regulatory concerns about PD-1 blockade in a different myeloma trial. In addition, comparison between effects of PD-1 and PD-L1 blockade is based on patients treated in different clinical studies and not as a part of a prospective randomized clinical trial directly comparing PD-1 and PD-L1 blockade. Such a clinical trial may now be feasible in malignancies wherein both PD-1 and PD-L1 blockade are now clinically approved and would be useful to dissect biologic differences between these therapies in humans.

The finding that PD-L1 blockade leads to rapid activation of inflammatory signatures on human myeloid cells in vivo suggests that the PD-L1 axis may be an important regulator of myeloid inflammation and impact emergency myelopoiesis and trained immunity in the clinic. While studies in human subjects described here are mostly correlative, a possible role of PD-L1 axis in regulating myeloid inflammation is also supported by emerging data from murine models, which is consistent with our studies. Engaging these pathways may be important for improving combination therapies with PD-L1 blockade, particularly harnessing the afferent arm of the cancer immunity cycle. Finally, differences in pharmacodynamic effects of PD-1 and PD-L1 blockade, as shown here, also have important implications for optimal combinations in the clinic, which may differ between these targets.

\section{Methods}

Patients and samples. For studies comparing genomic signatures of PD-1 and PD-L1 blockade, blood samples were obtained before and after 1 cycle of therapy from patients with advanced non-small cell lung cancer undergoing therapy with anti-PD-1 (nivolumab) (13) or anti-PD-L1 (atezolizumab).

Study design and monitoring. In the pilot study of atezolizumab in AMM (NCT02788843), patients were eligible if they met criteria for AMM based on BM clonal plasmacytosis of $>10 \%$ and/or levels 

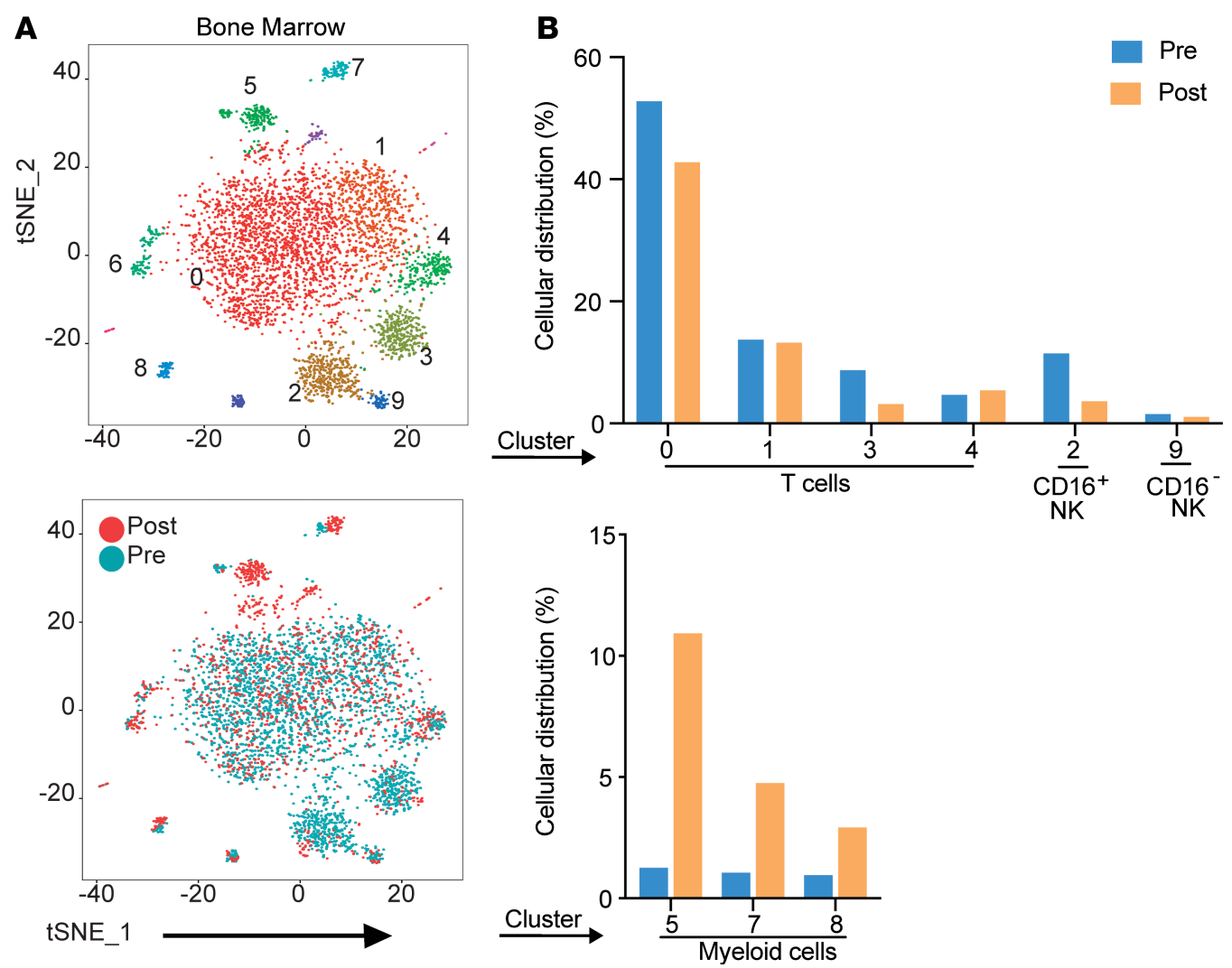

Figure 7. Changes in monocytes and effector T cells in BM following therapy with anti-PD-L1 in AMM by single cell RNA sequencing. BM was collected from AMM patient before and after 2 cycles ( 6 weeks) of therapy with atezolizumab. CD138- BM cells obtained before therapy and 6 weeks after therapy were characterized using sc-RNA-Seq. (A) t-SNE plot with 9 distinct populations determined by unsupervised clustering. Figure also shows distribution of the immune cells from before therapy BM (Pre) and after therapy BM (Post). (B) Percent of immune cells from before therapy (Pre) and after atezolizumab therapy (Post) within the clusters shown in $\mathbf{A}$.

of monoclonal immunoglobulin $>3 \mathrm{~g} / \mathrm{dL}$. Patients were also required to have an abnormal serum free light chain ratio (but $<100$ ) and absence of end organ damage based on CRAB criteria (hypercalcemia, renal dysfunction, anemia, bone disease), $<60 \%$ BM plasma cells, and no more than 1 known focal lesion on MRI. Other key eligibility criteria included the presence of measurable disease and adequate hematologic and organ function. Patients with any prior therapy for plasma cell disorder and history of active autoimmune disease were excluded. All eligible patients received atezolizumab $1200 \mathrm{mg}$ i.v. every 3 weeks. Blood samples for immune monitoring were collected at baseline, C1D15, and then before each cycle of therapy while in the study. BM biopsies were planned at baseline and after completion of 2 cycles of therapy.

Gene expression profiling of purified T cells and monocytes. Gene expression profiling of purified monocytes and $\mathrm{T}$ cells was performed as previously described (13). Briefly, CD14 ${ }^{+}$monocytes cells were isolated from PBMCs using immunomagnetic separation with anti-human CD14 microbeads (Miltenyi Biotec, 130-050-21), and T cells were isolated with human Pan-T cell isolation kit (Miltenyi Biotec, 130-096-535) following manufacturer instructions. RNA isolated from purified cells was analyzed using Affymetrix GeneChip Human Transcriptome Array (v2.0) as described (13).

Mass cytometry. Peripheral blood and BM mononuclear cells were immunophenotypically characterized using mass cytometry as described (25). The panel of antibodies used is shown in Supplemental Table 5. Data were acquired on Helios instrument (Fluidigm Sciences Inc.) and analyzed using Cytobank software (Cytobank Inc., Fluidigm).

$s c-R N A-s e q$. sc-RNA-seq of peripheral blood or BM mononuclear cells was performed using the 10× Genomics platform chromium single cell 3' kit following manufacturer's protocol as described $(14,26)$. Libraries were sequenced. Reads were aligned, filtered, deduplicated, and converted into a digital count 
A
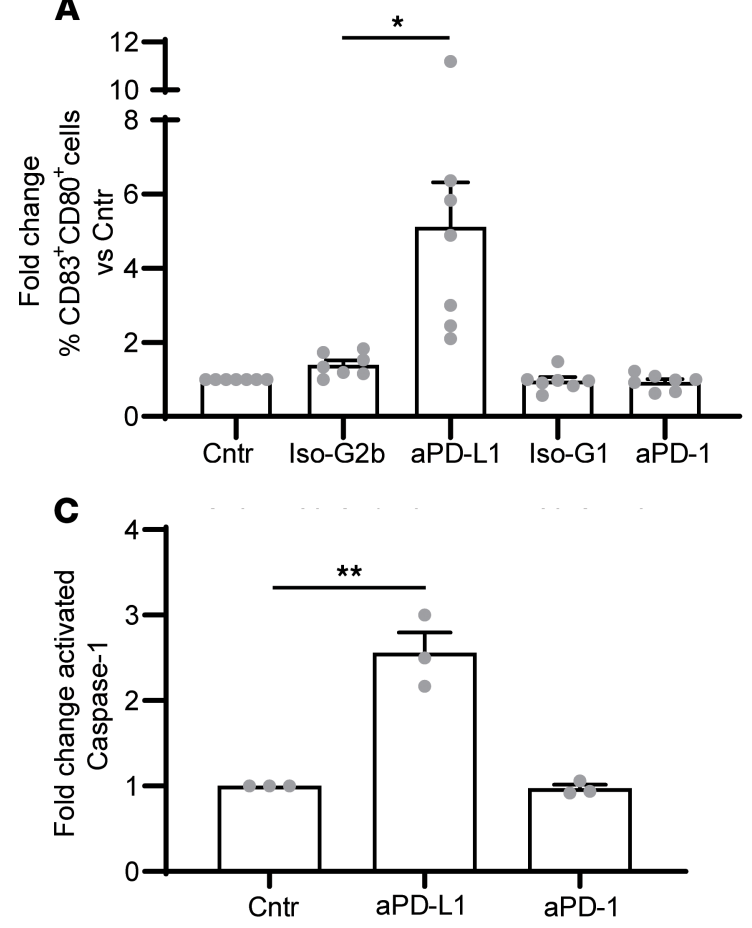

B
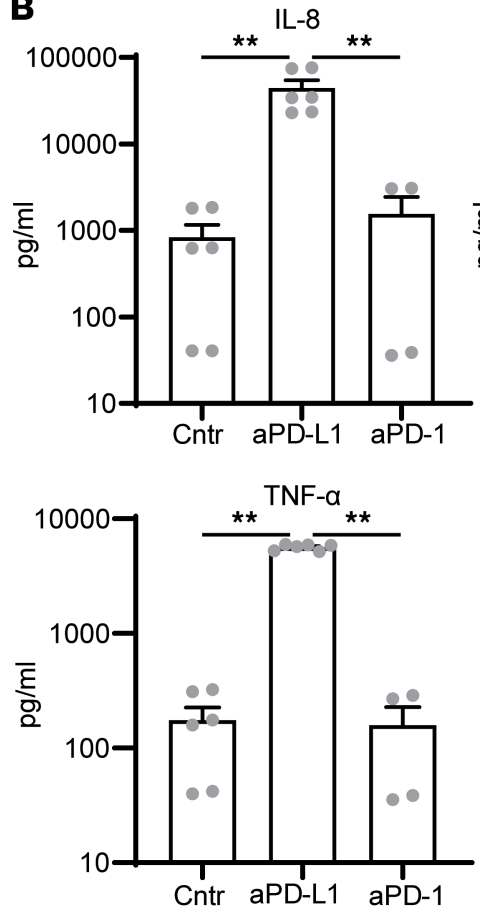

IL-6
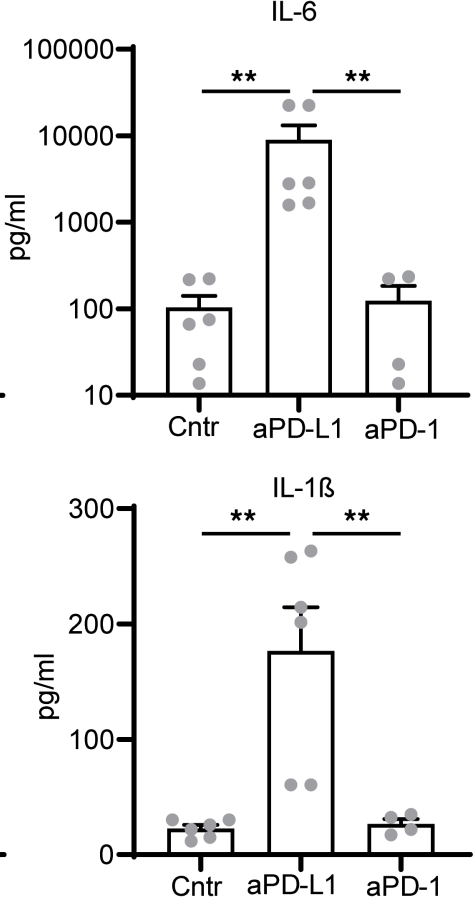

D
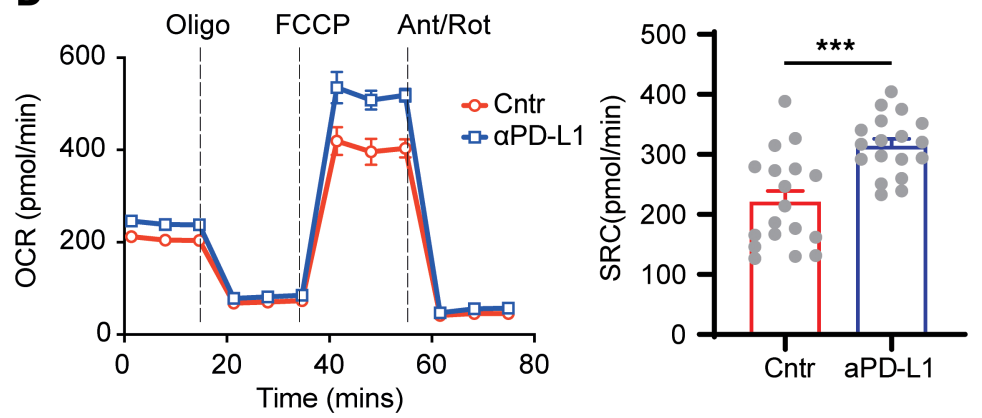

Figure 8. PD-L1 blockade leads to functional changes in DCs. Immature Mo-DCs generated from healthy blood donors were either left untreated (control, Cntr) or treated with either anti-PD-L1 antibody $(200 \mu \mathrm{g} / \mathrm{mL})$, anti-PD-1 antibody $(200 \mu \mathrm{g} / \mathrm{mL})$, or their respective isotype control antibodies (Ig-C2b and $\mathrm{Ig}-\mathrm{C} 1)$ at $200 \mu \mathrm{g} / \mathrm{mL}$ or CD40L ( $250 \mathrm{ng} / \mathrm{mL})$. Culture supernatants were analyzed for changes in cytokines using Luminex assay. Representative data from 7 healthy donors (HDs). (A) DC maturation following treatment with either anti-PD-L1, anti-PD-1, or isotype control. Figure shows fold change in CD83 and CD80 double-positive DCs compared with untreated cells. (B) Changes in secreted IL-8, IL-6, TNF- $\alpha$, and IL-1 $\beta$ following treatment with anti-PD-L1 or anti-PD-1. (C) Treatment with anti-PD-L1 leads to early activation of caspase-1. Fold change of activated caspase-1 in immature Mo-DCs following treatment with anti-PD-L1 or anti-PD-1 for 4 hours. Figure shows fold change compared with untreated cells (Cntr). (D) Changes in respiratory capacity of DCs following treatment with anti-PD-L1. Immature Mo-DCs ( $n=3 \mathrm{HDs}$ ) were either left untreated (control; Cntr) or were treated with anti-PD-L1 (200 $\mu \mathrm{g} / \mathrm{mL}$ for 3 hours), and their spare respiratory capacity was analyzed using Seahorse XFe96 analyzer. Basal, coupled, maximal, and spare respiratory capacities were analyzed. Line graph shows data from a representative patient. Bar graph on the right shows data from all 3 different donors (mean \pm SEM). ( $P<$ $0.05,{ }^{* *} P<0.01,{ }^{* *} P<0.001 ; \mathbf{A}, \mathbf{C}, \mathbf{D}$ used Mann-Whitney U test, and $\mathbf{B}$ used Kruskal Wallis test).

matrix using Cell Ranger 1.2 (10× Genomics). All downstream quality control and analyses were performed using Seurat (27). Cells with $\geq 200$ expressed genes were used for analysis.

For analysis of sc-RNA-seq from lung cancer patients treated with either anti-PD-1 or PD-L1, pre- and posttreatment samples for each patient were merged, and gene expression for each cell was log-normalized to total expression per cell. To reduce noise due to batch effects and interpatient heterogeneity, each patient was aligned and integrated with all other patients receiving the same treatment (anti-PD-L1, $n=3$; anti-PD-1, $n=4$ ) via canonical correlation analysis (CCA) using the Seurat FindIntegrationAnchors and IntegrateData functions. Gene expression data were then scaled such that each gene had a mean expression of 0 and a variance of 1 across all cells using the ScaleData function, and principal component analysis was performed using the RunPCA function. Data were visualized in 2 

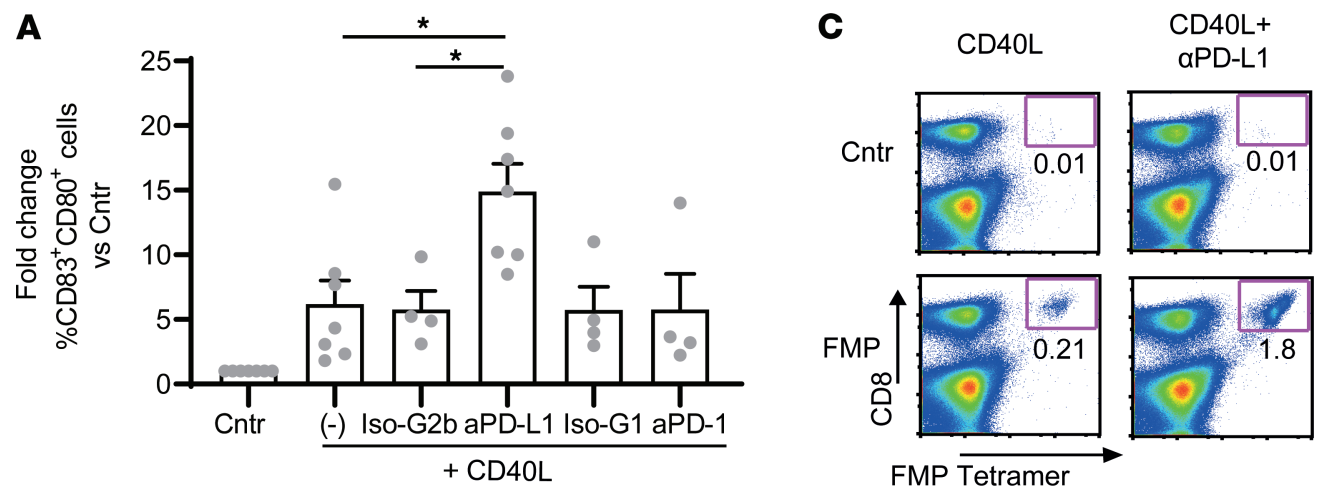
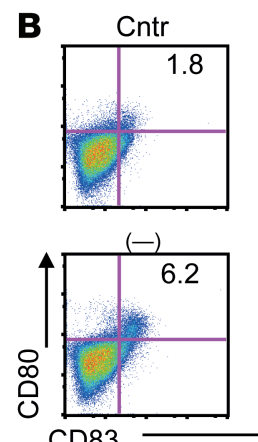

CD83

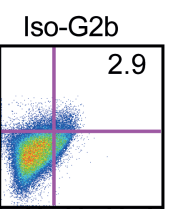

Iso-G2b

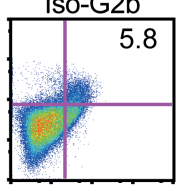

aPD-L1

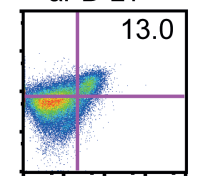

aPD-L1

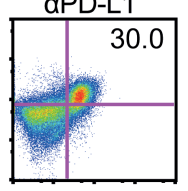

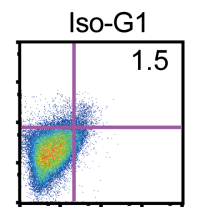

Iso-G1

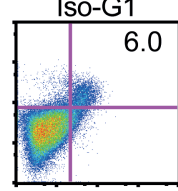

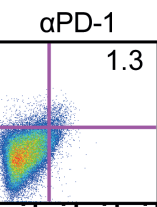

aPD-1

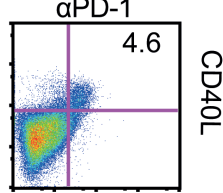

Figure 9. PD-L1 blockade synergizes with CD40L to improve antigen-specific T cell expansion. Immature Mo-DCs generated from healthy blood donors were either left untreated (control, Cntr) or treated with CD4OL $(250 \mathrm{ng} / \mathrm{mL}$ ) alone $(-)$ or with anti-PD-L1 antibody $(200 \mu \mathrm{g} / \mathrm{mL})$, anti-PD-1 antibody $(200 \mu \mathrm{g} / \mathrm{mL})$, or their respective isotype control antibodies (Ig-C2b and Ig-G1) at $200 \mu \mathrm{g} / \mathrm{mL}$. (A) Anti-PD-L1 treatment synergizes with CD40L to improve DC maturation. Figure shows fold change in DC maturation (assessed by increase in CD83 and CD80 double-positive cells) compared with control cells. (B) Representative data from one donor showing increased DC maturation with concurrent treatment with CD40L and PD-L1. (C) Immature Mo-DCs (HLA-A2.1+) were stimulated with CD40L alone or CD40L plus anti-PD-L1 antibody. After overnight culture, DCs were loaded with HLA-A2.1-specific influenza matrix peptide (FMP) at $0.1 \mu \mathrm{g} / \mathrm{mL}$ and used to stimulate autologous T cells. After 10-12 days of DC-T cell coculture, expansion of influenza-specific T cells was analyzed using FMP-specific tetramer. ( ${ }^{*} P<0.05$, Kruskal Wallis test).

dimensions using uniform manifold approximation and projection (UMAP) based on the first 20 principal components. Significant DEGs were identified by the Wilcoxon rank-sum test with a Bonferroni's correction $(P<0.05)$. Cluster identity was determined by inspection of canonical marker genes (e.g., CD14 for monocytes), and identity was confirmed by automated cell type determination with SingleR (https://bioconductor.org/packages/release/bioc/html/SingleR.html) via comparison with the Human Primary Cell Atlas (http://biogps.org/dataset/BDS_00013/primary-cell-atlas). Pathway analysis was performed using Gene Set Enrichment Analysis (GSEA) software (https://www.gsea-msigdb. org/gsea/index.jsp) and the Molecular Signature Database (MSigDB; https://www.gsea-msigdb.org/ $\mathrm{gsea} / \mathrm{msigdb} /$ index.jsp) from the Broad Institute.

Whole transcriptome analysis. Gene expression profiles from monocytes and $\mathrm{T}$ cells before and after antiPD-1 and anti-PD-L1 treatment were obtained using Affymetrix Human Transcriptome Array 2.0, and gene-level signal intensities were used for subsequent analysis. Preprocessing and normalization of data sets were carried out by Affymetrix Expression Console using gene level Signal Space Transformation-Robust Multiarray Average (SST-RMA) normalization. All downstream analyses were conducted using R and Bioconductor (28). The "limma" package was used for differential gene expression (29). GSEA was performed using Metacore (https://portal.genego.com) and MSigDB (Molecular Signatures Database v6).

Detection of antigen-specific T cells. In order to detect SOX2-specific T cells, PBMCs were stimulated for 48 hours with SOX2 peptide library as described (10). Following stimulation, the presence of T cell activation was determined based on the detection of IP-10 in the culture supernatant by Luminex.

Detection of plasma cytokines. Plasma samples were used for the detection of a panel of 38 cytokines/chemokines using the Milliplex MAP Human Cytokine/Chemokine Magnetic Bead Panel kit 
(HCYTMAG-60K-PX38; Millipore Sigma) as described (13). xPONENT software (Luminex Corp.) was used to detect, quantitate, and analyze the samples on the Luminex 100 instrument. Levels of IL-18 were analyzed using an Elisa kit (R\&D Systems).

Generation of Mo-DCs. Purified CD14 $4^{+}$monocytes were cultured in 1\% plasma in the presence of IL-4 (25 ng/mL; R\&D Systems) and GM-CSF (20 ng/mL sagramostim [Leukine]; Genzyme) to yield Mo-DCs. Immature Mo-DCs were used to study the effects of anti-PD-L1 or anti-PD-1 antibodies. DCs were cultured with anti-PD-L1 (clone 29E.2A3; BioLegend), anti-PD-1 (clone EH12.2H7; BioLegend) or their respective isotype control antibodies (IgG2b and IgG1; BioLegend) (200 $\mu \mathrm{g} / \mathrm{mL})$. For some experiments, immature Mo-DCs were cultured with CD40L (250 ng/mL; R\&D Systems).

Effects of PD-L1 blockade on BM myeloid cells. Bone marrow mononuclear cells (BMMNCs) were treated with atezolizumab (200 $\mu \mathrm{g} / \mathrm{mL})$ every 24 hours for 48 hours or left untreated. Following incubation, sam-

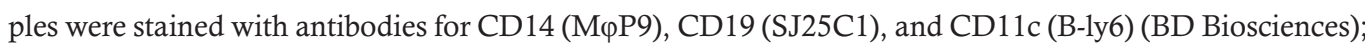
BDCA3 (AD5-14H12, Miltenyi Biotec); and PD-L1 (29E.2A3), CD40 (5C3), CD16 (3G8), and HLA-DR (L243) (BioLegend).

Antigen-specific $T$ cell stimulation. For some experiments, immature Mo-DCs differentiated from HLA A2. $1^{+}$donors $(n=4)$ were stimulated with CD40L $(250 \mathrm{ng} / \mathrm{mL})$ in the presence or absence of anti-PD-L1 $(200 \mu \mathrm{g} / \mathrm{mL})$. After overnight culture, DCs were loaded with HLA A2.1-restricted FluMP (sequence GILGFVFTL) at $0.1 \mu \mathrm{g} / \mathrm{mL}$ for 2 hours. Flu-MP-loaded DCs were then used to stimulate autologous $\mathrm{T}$ cells at a DC/T cell ratio 1:30 in the presence of IL-2 (10 U/mL). Flow cytometry analysis was performed to detect the presence of Flu-MP-specific CD8 ${ }^{+} \mathrm{T}$ cells using MHC tetramers (MBL International).

Detection of caspase-1 activation. Immature Mo-DCs were treated with anti-PD-L1 (200 $\mu \mathrm{g} / \mathrm{mL})$, antiPD-1 $(200 \mu \mathrm{g} / \mathrm{mL})$, or left untreated for 4 hours. Activation of caspase-1 was assayed with the FAMYVAD-FMK Caspase-1 Detection Kit (Cell Technology Inc). FAM-YVAD-FMK was added to the culture 1 hour before the end of culture period, following manufacturer protocol; washed twice with Caspase-1 kit wash buffer; and detected using flow cytometry.

Measurement of oxygen consumption and spare respiratory capacity. Basal, maximal, and coupled oxygen consumption rates were measured in a Mito stress assay using a Seahorse extracellular flux (XFe96) analyzer. Immature Mo-DCs were treated with anti-PD-L1 (200 $\mu \mathrm{g} / \mathrm{mL})$ or left untreated. After 3 hours, DCs were harvested, washed $1 \times$ with PBS, and plated at 200,000 cells per well in 5-8 replicates on Cell-Tak- precoated (Becton Dickinson) 96-well plates custom designed for XFe96 analysis. Oxygen consumption rate was evaluated over time with sequential injection of oligomycin (MilliporeSigma, catalog 495455; final concentration $2.5 \mu \mathrm{M}$ ), carbonyl cyanide p-trifluoro-methoxyphenyl hydrazone (FCCP; Enzo Life Sciences, BML-CM120-0010; final concentration $0.5 \mu \mathrm{M}$ ), and antimycin (Ant; MilliporeSigma, A8674) or rotenone (Rot; MilliporeSigma, R8875; final concentration $2 \mu \mathrm{M}$ each). Spare respiratory capacity was calculated as the difference between maximal and basal respiration.

Statistics. Data from individual cohorts were compared using GraphPad analysis software. Paired 2-tailed $t$ tests and nonparametric tests were used to analyze the data with significance set to $P<0.05$ and Bonferroni's to correct for multiple comparisons.

Study approval. The clinical trial was approved by the Yale University IRB and monitored by data safety monitoring committee at Yale Cancer Center. All specimens were collected following informed consent under institutional IRB guidelines at Emory and Yale universities.

\section{Author contributions}

NB supervised the clinical trial. FC, RD, and AD designed and performed experiments. M. Samur and SM performed bioinformatic analysis. SNG, NN, TLP, MLX, TA, NG, AN, and HJC performed clinical research. JKB, KP, RB, LZ, AR, and M. Shanmugam performed some experiments. MVD and KMD designed and performed oversight to entire project. All authors performed data analysis and approved the final manuscript.

\section{Acknowledgments}

This work is supported, in part, by funds from NIH/NCI (NCI CA197603 to MVD, CA238471 to KMD, CA208328 to MS, and P30CA138292 to Winship Shared Resources). 
Address correspondence to: Madhav V. Dhodapkar or Kavita M. Dhodapkar, 1760 Haygood Drive, Atlanta, Georgia 30322, USA. Phone: 404.778.1900; Email: madhav.v.dhodapkar@emory.edu (MVD); kavita.dhodapkar@emory.edu (KMD).

1. Zou W, Wolchok JD, Chen L. PD-L1 (B7-H1) and PD-1 pathway blockade for cancer therapy: Mechanisms, response biomarkers, and combinations. Sci Transl Med. 2016;8(328):328rv4.

2. Dong $\mathrm{H}$, et al. Costimulating aberrant $\mathrm{T}$ cell responses by $\mathrm{B} 7-\mathrm{H} 1$ autoantibodies in rheumatoid arthritis. J Clin Invest. 2003;111(3):363-370.

3. Azuma T, Yao S, Zhu G, Flies AS, Flies SJ, Chen L. B7-H1 is a ubiquitous antiapoptotic receptor on cancer cells. Blood. 2008;111(7):3635-3643.

4. Curiel TJ, et al. Blockade of B7-H1 improves myeloid dendritic cell-mediated antitumor immunity. Nat Med. 2003;9(5):562-567.

5. Lin H, et al. Host expression of PD-L1 determines efficacy of PD-L1 pathway blockade-mediated tumor regression. J Clin Invest. 2018;128(2):805-815.

6. Tang H, et al. PD-L1 on host cells is essential for PD-L1 blockade-mediated tumor regression. J Clin Invest. 2018;128(2):580-588.

7. Dhodapkar MV. MGUS to myeloma: a mysterious gammopathy of underexplored significance. Blood. 2016;128(23):2599-2606

8. Lesokhin AM, et al. Nivolumab in Patients With Relapsed or Refractory Hematologic Malignancy: Preliminary Results of a Phase Ib Study. J Clin Oncol. 2016;34(23):2698-2704.

9. Dhodapkar MV, Krasovsky J, Osman K, Geller MD. Vigorous premalignancy-specific effector T cell response in the bone marrow of patients with monoclonal gammopathy. J Exp Med. 2003;198(11):1753-1757.

10. Spisek R, et al. Frequent and specific immunity to the embryonal stem cell-associated antigen SOX2 in patients with monoclonal gammopathy. J Exp Med. 2007;204(4):831-840.

11. Dhodapkar MV, et al. Prospective analysis of antigen-specific immunity, stem-cell antigens, and immune checkpoints in monoclonal gammopathy. Blood. 2015;126(22):2475-2478.

12. Paiva B, et al. PD-L1/PD-1 presence in the tumor microenvironment and activity of PD-1 blockade in multiple myeloma. Leukemia. 2015;29(10):2110-2113.

13. Das R, et al. Combination therapy with anti-CTLA-4 and anti-PD-1 leads to distinct immunologic changes in vivo. J Immunol. 2015;194(3):950-959.

14. Das R, et al. Early B cell changes predict autoimmunity following combination immune checkpoint blockade. J Clin Invest. 2018;128(2):715-720.

15. Schoenberger SP, Toes RE, van der Voort EI, Offringa R, Melief CJ. T-cell help for cytotoxic T lymphocytes is mediated by CD40-CD40L interactions. Nature. 1998;393(6684):480-483.

16. Hartley GP, Chow L, Ammons DT, Wheat WH, Dow SW. Programmed Cell Death Ligand 1 (PD-L1) Signaling Regulates Macrophage Proliferation and Activation. Cancer Immunol Res. 2018;6(10):1260-1273.

17. Herbst RS, et al. Predictive correlates of response to the anti-PD-L1 antibody MPDL3280A in cancer patients. Nature. 2014;515(7528):563-567.

18. Tartey S, Kanneganti TD. Differential role of the NLRP3 inflammasome in infection and tumorigenesis. Immunology. 2019;156(4):329-338.

19. Ghiringhelli F, et al. Activation of the NLRP3 inflammasome in dendritic cells induces IL-1beta-dependent adaptive immunity against tumors. Nat Med. 2009;15(10):1170-1178

20. McDermott DF, et al. Clinical activity and molecular correlates of response to atezolizumab alone or in combination with bevacizumab versus sunitinib in renal cell carcinoma. Nat Med. 2018;24(6):749-757.

21. Lo Russo G, et al. Antibody-Fc/FcR Interaction on Macrophages as a Mechanism for Hyperprogressive Disease in Non-small Cell Lung Cancer Subsequent to PD-1/PD-L1 Blockade. Clin Cancer Res. 2019;25(3):989-999.

22. Kukreja A, et al. Enhancement of clonogenicity of human multiple myeloma by dendritic cells. J Exp Med. 2006;203(8):1859-1865.

23. Zheng Y, et al. Macrophages are an abundant component of myeloma microenvironment and protect myeloma cells from chemotherapy drug-induced apoptosis. Blood. 2009;114(17):3625-3628.

24. Bailur JK, et al. Early alterations in stem-like/resident T cells, innate and myeloid cells in the bone marrow in preneoplastic gammopathy. JCI Insight. 2019;5:127807.

25. Boddupalli CS, et al. Interlesional diversity of $\mathrm{T}$ cell receptors in melanoma with immune checkpoints enriched in tissue-resident memory T cells. JCI Insight. 2016;1(21):e88955.

26. Kini Bailur J, et al. Changes in bone marrow innate lymphoid cell subsets in monoclonal gammopathy: target for IMiD therapy. Blood Adv. 2017;1(25):2343-2347.

27. Satija R, Farrell JA, Gennert D, Schier AF, Regev A. Spatial reconstruction of single-cell gene expression data. Nat Biotechnol. 2015;33(5):495-502.

28. Huber W, et al. Orchestrating high-throughput genomic analysis with Bioconductor. Nat Methods. 2015;12(2):115-121.

29. Ritchie ME, et al. limma powers differential expression analyses for RNA-sequencing and microarray studies. Nucleic Acids Res. 2015;43(7):e47. 